\title{
Effect of Highly Active Antiretroviral Therapy (HAART) on Human Immunodeficiency Virus Disease Pathogenesis and Progression
}

\author{
K V Ramana* \\ Department of Microbiology, Prathima Institute of Medical Sciences, Karimnagar, Andhrapradesh \\ *Corresponding author: ramana_20021@rediffmail.com
}

Received March 31, 2014; Revised April 11, 2014; Accepted April 13, 2014

\begin{abstract}
Human immunodeficiency virus (HIV), the causative agent of Acquired Immunodeficiency Syndrome (AIDS) was discovered way back in 1983. More than three decades since its discovery, HIV infection diagnosis, treatment and management has been a big challenge to the medical field. HIV infection is attributed to cause compromised T-cell and B-cell immunity, promote different malignancies and the infected individuals are at risk of acquiring other viral, bacterial, fungal and parasitic infections. HIV disease progression was found different in infected population, where few HIV infected patients develop AIDS in less than 5 years and are called as early progressors, HIV infected population in whom development of AIDS is slow and may remain normal up to 10 years are termed as late progressors and patients in whom the symptoms of AIDS are not seen even after 10-15 years are labeled as long term non-progressors. The variability in disease progression is not completely understood. After the discovery of HAART, the mortality of the HIV infected population has significantly reduced but the morbidity attributed to HAART has remained as a serious concern. From being a life threatening infection HIV has now become a chronic infection, where patients live their natural life years with the HAART therapy. Among the most significant changes in HIV infection and pathogenesis is the development of non-infectious complications attributed to the HIV infection, HAART therapy, other demographic factors and co-morbidities. Other factors that influence the disease pathogenesis and progression include chronic immune activation, drug resistance and toxic side effects of HAART therapy. Among this Immune activation plays a key role in the pathogenesis and progression of HIV infection.
\end{abstract}

Keywords: HIV disease pathogenesis, HAART, immune activation, non-AIDS related complications

Cite This Article: K V Ramana, "Effect of Highly Active Antiretroviral Therapy (HAART) on Human Immunodeficiency Virus Disease Pathogenesis and Progression.” American Journal of Public Health Research, vol. 2, no. 3 (2014): 68-74. doi: 10.12691/ajphr-2-3-1.

\section{Introduction}

Human immunodeficiency virus replicates in TCD4+ cells and results in the depletion of cells which in turn is responsible for gradual decline in the $\mathrm{T}$ cell immunity. HIV viral replication results in the production of viral particles which are responsible for the development of chronic inflammation that contributes hugely to the disease pathogenesis and progression towards AIDS and death [1]. Introduction of HAART has significantly reduced the morbidity and mortality in HIV infected patients resulting in extended life on par with HIV non infected individuals [2]. Of late research has revealed that HIV infected individuals are at greater risks developing non infectious complications (Liver disease, cardiovascular disease (CVD)) that may precipitate with the initiation of HAART [3]. With the easy availability and affordability of HAART, the focus now is on developing effective strategies to monitor HIV disease progression and treatment response [4,5]. Though pathogenesis of HIV is complex and a lot remains to be understood, studies thus far have been successful in applying the available knowledge to reduce the morbidity and mortality. Early initiation of HAART has been responsible for effective control of viral replication as evidenced by the undetectable amount of viral load in patients treated with HAART. From being an infection that was potentially fatal, HIV infection has now evolved in to a chronic disease. Initiation of HAART at the right time and close monitoring of the patients during therapy can significantly bring down the morbidity and mortality rates and improve the quality of lives [6]. Other important aspects of HIV disease and HAART therapy management is the identification of co-morbidities, drug toxicities, drug resistance and treatment compliance. HIV infection remains permanent and the virus gradually spreads to all parts of the body infecting TCD4+ cells of various organs. Depending on the rate of depletion of TCD4+ cells, the infected patients are at risk of developing various opportunistic microbial infections and cancers (pneumonia, tuberculosis, parasitic diarrhoea, Kaposi's sarcoma and 
non-Hodgkin's lymphoma) and most of the patients develop advanced disease and succumb $[7,8]$. Reports of HIV infected population who do not show significant depletion of TCD4+ cell counts and remain healthy have paved the way for further research that can be useful in the development of vaccine and functional cure of HIV. It still remains to be understood why uninfected TCD4+ cells and other immune cells die or manage to control the HIV infection. Studies have confirmed the role of chronic immune activation in the HIV disease pathogenesis and progression $[9,10]$. HAART, though has been instrumental in the reduction of inflammation, the major cause of concern that remains is the toxic effects of antiviral drugs. Antiretroviral drugs are selected based on the type of sideeffects they produce, drug-drug interactions and the potency [3]. A successful antiviral agent is described as the one that brings about decrease in HIV/RNA plasma viral load. Antiretroviral drugs fall in to six categories: non-nucleoside reverse transcriptase inhibitors (NNRTI's) including Nevirapine, Efavirenz and Etravirine, Nucleoside and nucleotide reverse transcriptase inhibitors (NRTI's) that include Zidovudine, Abacavir, Lamivudine, Stavudine, Tenofovir and Emtricitabine, Integrase inhibitors such as Raltegravir, Protease inhibitorssaquinavir, Lopinavir, Fosamprenavir, Darunavir and atazanavir, CCR5-Inhibitor including Maraviroc and Fusion inhibitor-Enfuvirtide [3,6].

\section{Management of Non-Infectious Complications}

Virological (HIV/RNA viral load), immunological (TCD4+ cell counts), haematological (Haemoglobin (HB\%, total leukocyte count (TLC), Erythrocyte sedimentation rate (ESR), C-reactive protein (CRP) and others) and biochemical markers (Albumin, Globulin, cholesterol and others) have been shown to have great potential in the management of HIV disease and treatment response. Studies in the past have also noted that the biological markers like neopterin, $\beta-2$ microglobulin, Dehydroepiandrosterone (DHEAS) and many others may be useful in the management of HIV infected individuals and those on antiretroviral therapy. Tumor necrosis factor$\alpha$ (TNF- $\alpha$ ), Interleukins (IL-2, IL-6, and IL-10) and other cytokines have been demonstrated to have significant role in the monitoring of HIV disease and HAART therapy [11-16]. Since huge population of HIV infected individuals live in poor and economically weak third world nations, which have financial limitations to acquire necessary equipment, research is now concentrated on evaluation of cheaper and easily performed alternate markers that can be used to regularly monitor HIV infected population and antiretroviral therapy response.

Considering the adverse effects of HAART, previous studies have suggested that HIV infected patients should be screened for haematological abnormalities (Anaemia) before initiation of antiretroviral therapy [11]. In a study that we have performed recently on the occurrence and usability of Haemoglobin \% and Erythrocyte sedimentation rate (ESR) revealed that there was anaemia

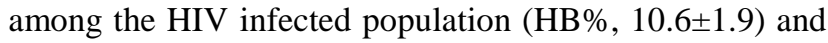
after initiation of HAART the levels of haemoglobin have increased $(\mathrm{HB} \%, 10.8 \pm 2.2)$ indicating better prognosis. Evaluation of ESR after 4-6 months of HAART therapy revealed a mild reduction in the levels of ESR also considered as better prognosis (ESR before HAART (16.3 \pm 8.9 and ESR after initiation of HAART $(13 \pm 7.2)$ [13]. Worsening of anaemia and increase of inflammatory markers including ESR after initiation of HAART therapy should be considered as a cause of serious concern [13] Figure 1 and Figure 2. Our recent studies have evaluated the role of serum gamma-glutamyl transpeptidase (GGT), activities of lactate dehydrogenase (LDH) and their utility as alternate biological markers in the management of HIV infection and treatment response [14,15].

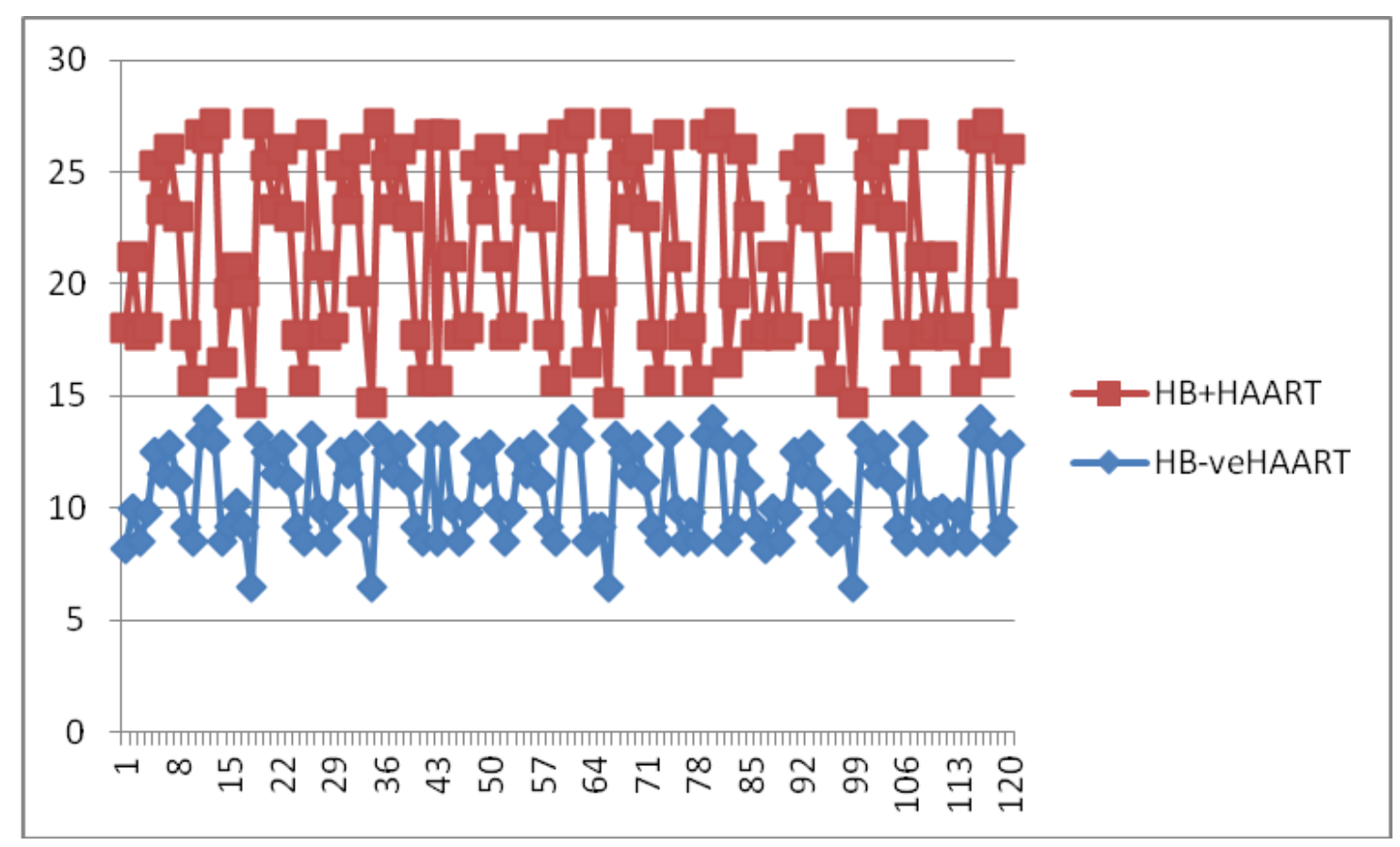

Figure 1. Hemoglobin [HB\%] before and after HAART 


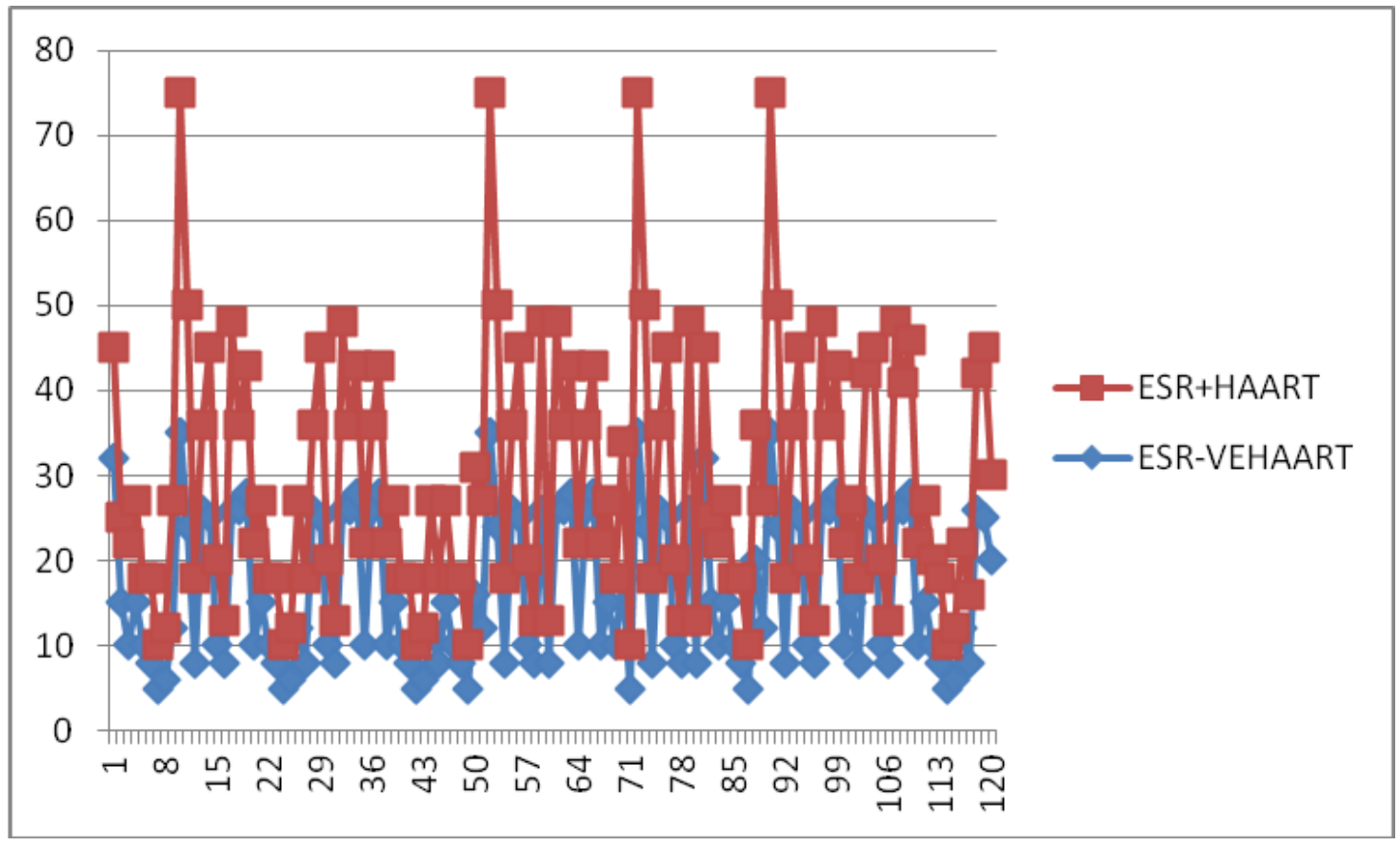

Figure 2. Erythrocyte Sedimentation Rate [ESR] before and after HAART

\section{HIV, HAART and Liver Disease}

HIV infected population are prone to liver disease as evidenced from the available literature $[3,14]$. This is further worsened by the initiation of HAART as the toxic effects of antiretroviral therapy play a key role in the pathogenesis and progression of HIV disease. HIV infection by itself may contribute to hepatic and biliary tract abnormalities including hepatomegaly, liver fibrosis, liver cirrhosis, hepatic/biliray steatosis and elevated liver enzymes. Hepatotoxicity, hyperlipidemia, hyperglycemia, and lactic acidosis are some of the adverse conditions of HAART therapy. Hepatotoxicity can influence HIV-1 treatment and can cause an increase in morbidity and mortality. Protease Inhibitors (PIs), nonnucleoside reverse transcriptase inhibitors (NNRTIs), and the chemokine receptor 5 (CCR5) antagonist, maraviroc, can be hepatotoxic with asymptomatic elevations in liver enzymes, liver dysfunction and liver failure. Co-infections with other hepatocellular viral infections like Hepatitis B virus and hepatitis $\mathrm{C}$ virus may further be responsible to liver related severe morbidity and mortality [17]. Alcoholism, lifestyle diseases like obesity, hypertension and diabetes mellitus may act as co-factors in worsening the liver disease. Non-antiretroviral drugs, used in the treatment of secondary bacterial, viral, parasitic and fungal infections could also contribute to the liver dysfunction. Although some previous studies have noted the improvement in the liver performance after initiation of HAART, much still remains to be known about the possible toxic effects of long term antiretroviral therapy [18]. HAART therapy involves use of at least two nucleoside analogue reverse transcriptase inhibitors (NRTI); zidovudine (ZDV), zalcitabine (ddC), didanosine (ddI), lamivudine (3TC) or stavudine (d4T) combined with a protease inhibitor (PI) or with a non-nucleoside analogue reverse transcriptase inhibitor (NNRTI) such as efavirenz (EFV). Long-term side effects associated with the use of NRTI's and their ability to inhibit polymerase, the enzyme responsible for replication of mitochondrial DNA (mt DNA). Depletion of mtDNA in the liver may manifest as micro vesicular steatosis, steatohepatitis or liver failure [18]. Studies in the recent past have evaluated the utility of liver enzymes (Alanine aminotransferase (ALT), Aspartate aminotransferase (AST) and gammaglutamyl transpeptidase) in the management of HIV seropositive patients receiving HAART [14].

\section{HIV, HAART and Cardiovascular Disease}

HIV infection is associated with increased cardiovascular risk as evidenced by the available literatures that have shown elevated levels of cholesterol, triglycerides and other lipid abnormalities [19]. Coronary arterial disease (CAD) is the leading cause of non-HIV-1related death in people infected with HIV-1 [3]. HIV-1 infection may be an independent risk factor for Cardio vascular disease (CVD), although there is not yet sufficient evidence to consider it a Coronary heart disease (CHD) risk equivalent. Factors responsible for cardiovascular risk among HIV-1 infected population may be related to the chronic inflammatory response, metabolic disorders (insulin resistance, dyslipidemia, abnormal fat distribution) and adverse effects of antiretroviral therapy. Antiretroviral therapy may prove beneficial in reducing the cardiovascular complications, but one should be cautious about the immune dysfunction, immune activation and severe inflammatory conditions produced by HAART $[20,21]$. Previous studies suggests that the nucleoside analogue, reverse transcriptase inhibitors (NRTIs) including stavudine, didanosine, and zidovudine may cause mitochondrial toxicity by inhibiting mitochondrial DNA polymerase in fat and other tissues and thus interfering with respiratory chain complexes which results in impaired fatty acid oxidation and intracellular accumulation of triglycerides and lactate (SREBPs), which can enter the systemic circulation. 
Protease inhibitors (PIs) inhibit maturation of sterol response element binding proteins, which affect intracellular fatty acid and glucose metabolism and adipocyte differentiation. The PIs also down-regulate peroxisome proliferator-activated receptor gamma (PPAR $\gamma$ ), an important nuclear transcription factor that is affected by SREBPs and is necessary for adipocyte differentiation and function and fatty acid metabolism. Lipodystrophy and dyslipidemia are also observed in acute HIV-1 infection and HAART naive patients, lending support to HIV viral role as well. Potential host risk factors include age, sex, life style, genetic predisposition, obesity and race or ethnicity. Both HIV-1 infection itself and antiretroviral therapy can cause and worsen lipid abnormalities. Management of dyslipidemia in the HIV-1infected population on HAART therapy requires awareness of the toxic effects of antiretroviral agents on lipid metabolism, including potential age, sex and race related effects [22]. Although the exact mechanism by which HIV infection triggers CVD is not fully understood a recent study has shown that in chronic HIV infection, despite HAART the increased expression of IL-1 $\beta$ on infected monocytes stimulate the excessive production of pro-inflammatory cytokine IL-6 [19].

\section{HIV, HAART and Immune Activation}

HIV infection, pathogenesis and disease progression is complex which involves both immune deficiency and deregulated immune-activation and remains different in various populations. HIV infects TCD4+ cells, compromises immunity and predisposes infected patients to various infectious, inflammatory and cancerous conditions. Studies in the past have elaborated on the role of HIV in the induction of chronic immune activation that plays a key role in the disease progression and pathogenesis [1]. Chronic immune activation is characterized by exhaustion of TCD4+ cells and TCD8+ cells and results in increased viral replication and viraemia. Immune activation in HIV infected patients' results with the release of pro inflammatory markers which are responsible for suppression of hemopoetic cells in the bone marrow and the development of blood cytopenias $[23,24]$. Studies have also noted that immune activation may result possibly involving two distinct pathways and both distinctively affect TCD4+ and TCD8+ cells [25]. Early HIV infection leads to the development of cytokine imbalance between the viral inductors (IL-6, IL-15, IL-1 $\beta$ and Tumour necrosis factor TNF- $\alpha$ )) and viral suppressors (IL-10 and type 1Interferons (IFN- $\alpha$ and IFN- $\beta$ )) that transforms in to an equilibrium where virus replication may be controlled/uncontrolled resulting in to variable disease progression [26,27]. Studies in the past have noted that initial infection of HIV resulted in the rise of cytokines like IFN- $\gamma$ and IL-12 associated with lower viral loads and appearance of elevated IL-7, IL-15 and lower TCD4+ cells with higher viral loads. The same study has noted that there was a rise in pro inflammatory cytokines like IL-2, IL-7, IL-15 and IL-21 that play a key role in improved immune defence against HIV proliferation [28]. A study by Wren et al revealed that appearance of IL-10 and IL-15 is associated with improved activity of anti-HIV natural killer cells (NK- cells) suggesting utility of these cytokines as prophylactic preparations/intervention [29]. Other studies have shown that a decrease in IL-2 and IFN- $\gamma$, simultaneous increase in IL-4 and IL-10 may be associated with compromised antigen-specific viral defence mechanism, increased lymphocytic apoptosis and decrease in TCD8+ cells [30, 31 and 32]. It has been observed that elevated activities of TCD8+ cells are instrumental in natural suppression of HIV infection as observed in long term non progressors (LTNP) and that decreased activation of TCD4+ cells and Th1 cells are associated with slow progression [33,34].

After the initiation of antiretroviral therapy, the activity of various cytokines and chemokines were elaborated by various working groups and found contrasting results $[35,36,37]$. Some studies have noted insignificant changes in the cytokines and other inflammatory markers (IL-6 and C-reactive protein) and other have revealed a decrease in IL-1 $\beta$, IL-6, IL-12, IFN- $\gamma$ and TNF- $\alpha$ after 4-6 months of HAART $[38,39,40]$.

In correlation with TCD4+ cells, it was noted that low counts were observed with increased cytokine levels of TNF- $\alpha$, IL-6, IL-1ra and IL-8 [41]. There are contrasting reports of IL-10 levels among patients receiving HAART, as some show reduction and others reveal raised activities $[42,43,44]$. This needs further studies as IL-10 is found to have therapeutic efficacy. A recent study by Cozzi-Lepri et al have revealed that an interruption in the treatment was associated with increased viral load, lowering TCD4+ cells and increased production of viral induction cytokines like IL-6, IL10 and TNF- $\alpha$, a consequence of immune activation and immunological deficiency [45]. A study on the utility of IL-18, a pro inflammatory cytokine marker has shown that a decrease in the levels of IL-18 was observed in patients receiving Antiretroviral therapy and an increase was noted among those not treated which were correlating well with viral loads [46,47]. Performance of Th17 cell types in the initiation and worsening of immune activation revealed increased production of IL-17, IL-1, IL-2, IL-21, IL-22 and TNF- $\alpha[48,49,50]$. It has been observed that the pro inflammatory activity induces gradual mucosal barrier/tissue damage and results in chronic generalized inflammation and disease progression [51]. Studies on the effect of age as a co-morbid condition on the HIV disease progression and antiretroviral therapy revealed that though HAART is beneficial in the viral suppression, there is a possibility of chronic immune activation that can result in age-related multi-organ morbidity [52]. Microbial translocation, presence of bacterial enzymes, antigens (lipopolysacharides) and toxic by products could precipitate immune activation and accelerate HIV disease progression [53]. Chronic immune activation in HIV infected population many contribute to the development of cancers by stimulating cell proliferation, production of reactive oxygen and nitrogen species, enhance secretion of pro carcinogenic cytokines and chemokines and related mechanisms [54,55]. Studies thus far have confirmed that chronic immune activation and elevated activity of inflammatory cell may contribute to increased mortality even in the presence of HAART and irrespective of TCD4+ cell counts [56]. A recent study has elaborated on the possible role of Treg cells (CD4CFOXP3+), a group of regulatory $\mathrm{T}$ cells in generation of immune activation during HIV infection [57]. Another recent observation has elaborated the 
possible reason behind the immune activation in chronically infected HIV patients and found that exposure of immune system to viral antigens and other microbial antigen could be responsible for continuous T-cell activation [58]. A recent research has revealed the influence of HIV over antigen presenting cells (APC's) to react with commensal bacteria could contribute to further immune activation and inflammation [59]. Immune activation mechanism studies in primary HIV infection (PHI) has shown that immune activation is not driven solely by impairment of mucosal barrier and microbial translocation (MT) but by viral replication as evidenced by expression of soluble TCD14+ cells and IL-1RA [60]. Recent research has identified the role of HLA-DR, Ki67 and CD38 expression on regulatory $\mathrm{T}$ cells (Tregs) including TCD4+,TCD25+, TCD127+ contribute to immune activation in HIV infected but treatment naive patients [61]. Read SW et al from their study have demonstrated the significance of leflunomide, an immunomodulator in reducing the immune activation in chronic HIV infected population [62]. Studies on simian immunodeficiency virus infection in sooty mangabeys have confirmed that immune activation plays a significant role in HIV disease progression, where SIV infected sooty mangabeys show no signs of disease progression and absence of immune activation even when viral replication is present [63]. Human studies have also noted that use of cyclosporine, an immune-modulator along with HAART has reduced the rapidity of $\mathrm{T}$ cell activation and cell death [64]. A recent study by Gilad Doitsh et al has revealed that immune activation and depletion of TCD4+ cells may be due to pyroptosis, an intense inflammatory reaction caused due to release of pro inflammatory cytokines occurring in activated $\mathrm{T}$ cells and cells surrounding such cells resulting in cell death [65]. Animal studies in the recent past have also elaborated on the use of pro-biotic and pre-biotic supplementation that help in immune reconstitution, better functioning of mucosal barriers and reduced fibrosis of lymphoid system [66].

\section{Future of HIV Disease}

Ever since its discovery, way back in 1983, HIV has always been a big challenge to the medical fraternity. Advancements in the diagnosis of HIV infection have come to a level where most of the centres are well equipped even in developing and economically constrained nations. After the introduction of HAART in 1995, the availability and affordability has improved globally. HAART though has been successful in reducing the mortality, the cause of concern is the long-term toxic consequences and the increase in non-AIDS related conditions in HIV infected population receiving HAART. Neurological complications, haematological and organ related malignancies, bone and connective tissue disorders, cardiovascular, renal and hepatic diseases are some of the serious non-AIDS events which are responsible for morbidity and mortality in HAART era $[67,68,69]$. From being a life threatening infectious disease, HIV has now become a chronic infectious disease having potential for severe morbidity and mortality if not properly managed [70,71,72]. Special HIV-care centres would be ideal for the management of HIV infected population, where physicians involved in the treatment would manage the patients both for AIDS related and non-AIDS related complications. Evaluation of co-morbidities, both infectious and non infectious, which may contribute to disease progression, is a matter of serious concern for better HIV disease management. Interventions including the anti-inflammatory drugs (sevelemer-anti-LPS, antiPD1 antibodies, anti IL-6 antibodies, anti IFN- $\alpha$ antibodies) and HIV cure concepts (Histone deacetylase inhibitors, disulfiram, CCR5-modified T cells and stem cells, IL-7, IL-15 therapy and methylation inhibitors) provide a route map and hope that may at last result in HIV functional cure [73,74,75]. Health care system must be acquainted with skilled and volunteering individuals who take the responsibility to identify HIV infected patients, provide access to therapy and continuously monitor treatment response and disease progression. It has also been noted that HIV care become even more complicated with ageing HIV infected population $[76,77,78]$.

\section{Conclusion}

Although HAART has revolutionised HIV care, factors contributing to chronic inflammation even in patients undergoing treatment, influence of co-morbidities, and antiviral drug toxicities and resistance on disease course are subjects that need extensive research for achieving better patient management in HIV seropositive patients receiving HAART. Early diagnosis of non-AIDS related complications, initiating treatment and management strategies and regular monitoring would benefit HIV patients to reduce the morbidity and increase quality of life and life expectancy.

\section{Acknowledgement}

I sincerely acknowledge the support of the management, Prathima Institute of Medical Sciences, Karimnagar.

\section{References}

[1] Deeks SG. Immune dysfunction, inflammation, and accelerated aging in patients on antiretroviral therapy. Top HIV Med 2009; 17:118-23.

[2] K V Ramana. Are we close enough to get rid of AIDS: Insights in to impact of Human Immunodeficiency virus (HIV) infection post Highly Active Antiretroviral therapy (HAART) Era. International Journal of Molecular Medical Science, 2013; 3(5): 25-29.

[3] K V Ramana and Ratna rao. Human Immunodeficiency Virus disease management in Highly Active Anti-Retroviral Therapy era: a comprehensive review Ann of Trop Med Public Health 2013; 6(1): 5-9.

[4] Ramana, K V. "Markers of HIV-1 Disease Progression and Treatment Response in Highly Active Antiretroviral Therapy (HAART) Era: A Review." American Journal of Infectious Diseases and Microbiology 1.5 (2013): 96-100.

[5] Ramana K V, Rao R. Noninfectious complications in HIV disease: Need for rational changes in HIV disease management in the highly active antiretroviral therapy era. Ann Trop Med Public Health 2013; 6: 383-5.

[6] Paul A Volberding, Steven G Deeks. Antiretroviral therapy and management of HIV infection. Lancet 2010; 376: 49-62.

[7] K. V. Ramana and S. K. Mohanty Opportunistic intestinal parasites and $\mathrm{TCD}^{+}$cell counts in human immunodeficiency virus seropositive patients. J Med Microbiol 2009. 58: 1664-1666. 
[8] Ramana K V. HIV Disease Management in the Highly Active Antiretroviral Therapy (HAART) Era. J Medical Microbiol Diagnosis 2012; 1:e101.

[9] Ascher MS and Sheppard HW: AIDS as immune system activation: A model for pathogenesis. Clin Exp Immunol 1988; 73(2):165-167.

[10] Hazenberg MD, Hamann D, Schuitemaker H, and Miedema F: T cell depletion in HIV-1 infection: how CD4 + T cells go out of stock. Nat Immunol 2000; 1(4): 285.

[11] Ramana K V, Jagadeeswhwara chary, Sabitha V, S K Mohanty, Ratna Rao. Role of Hematological and Alternate Markers in Human Immunodeficiency Virus Disease Progression. American Medical Journal 2010; 1 (2): 84-87.

[12] K V Ramana, V Sabitha, Ratna Rao. A Study of Alternate Biomarkers in HIV Disease and Evaluating their Efficacy in Predicting $\mathrm{T}$ CD4+ cell counts and Disease Progression in resource poor settings in Highly Active Antiretroviral Therapy (HAART) Era. Journal of Clinical and Diagnostic Research [serial online] 2013 July [cited: 2013 Jul 5]; 7:1332-1335.

[13] Kandi Venkataramana. A Study of Biological Markers in HIV Disease Progression and Management in the Highly Active Antiretroviral Therapy (HAART) Era, American Journal of Bioscience and Bioengineering. 2013; 1(2): 24-37.

[14] Ramana KV, Ratna Rao, Sabitha Abnormal Levels of $\gamma$-Glutamyl Transpeptidase (GGTP), ALT, AST in Human Immunodeficiency Virus-1(HIV-1) Infection. Biochem Physiol 2012; 1:101.

[15] Ramana K V, Rao R, Kandi S, Singh PA, Kumar VP. Elevated activities of serum lactate dehydrogenase in human immunodeficiency virus sero-positive patients in highly active antiretroviral therapy era. J Dr NTR Univ Health Sci 2013;2:162-6.

[16] Ramana KV, Ratna Rao, Sabitha, Venugopal B, Rafi MD, et al. Biochemical Parameters in Human Immunodeficiency Virus Disease Progression. J Medical Microbiol Diagnosis 2012; 1:103.

[17] K V Ramana etal, Seroprevalence of blood-borne viral infections in post HAART era at a tertiary care hospital in south India: A five year trend analysis (2008-2012) and a comprehensive review. British Journal of Medicine and Medical Research 2013; 3 ( 4) : 1929-1937.

[18] José Antonio Mata-Marín, Jesús Gaytán-Martínez, Bernardo Horacio Grados-Chavarría, José Luis Fuentes-Allen, Carla Ileana Arroyo-Anduiza and Alfredo Alfaro-Mejía. Correlation between HIV viral load and aminotransferases as liver damage markers in HIV infected naive patients: a concordance cross-sectional study. Virology Journal 2009, 6:181.

[19] Jalbert E, Crawford TQ, D’Antoni ML, Keating SM, Norris PJ, et al. IL-1B Enriched Monocytes Mount Massive IL-6 Responses to Common Inflammatory Triggers among Chronically HIV-1 Infected Adults on Stable Anti-Retroviral Therapy at Risk for Cardiovascular Disease. PLoS ONE 2013; 8(9): e75500.

[20] Gill VS, Lima VD, Zhang W, et al. Improved virological outcomes in British Columbia concomitant with decreasing incidence of HIV type 1 drug resistance detection. Clin Infect Dis 2010; 50: 98-105.

[21] The Antiretroviral Therapy Cohort Collaboration. Life expectancy of individuals on combination antiretroviral therapy in highincome countries: a collaborative analysis of 14 cohort studies. Lancet 2008; 372: 293-99.

[22] Dr Aberg Management of Dyslipidemia and Other Cardiovascular Risk Factors in HIV-Infected Patients: Case-based Review. Top HIV Med. 2006;14(4):134-139.

[23] $\mathrm{N}$ Vanker. The use of the full blood count and differential parameters to assess immune activation levels in asymptomatic, untreated HIV infection. S Afr Med J 2014; 104(1):45-48.

[24] Karen Ingrid Tasca, Sueli Aparecida Calvi and Lenice do Rosário de Souza. Immunovirological parameters and cytokines in HIV infection Rev Soc Bras Med Trop 2012; 45(6):663-669.

[25] Marta Catalfamo, Michele Di Mascio, Zonghui $\mathrm{Hu}$, Sharat Srinivasula, Vishakha Thaker, Joseph Adelsberger. HIV infection-associated immune activation occurs by two distinct pathways that differentially affect CD4 and CD8 T cells. PNAS 2008; 105 (50): 19851-19856.

[26] Keating SM, Jacobs ES, Norris PJ. Soluble mediators of inflammation in HIV and their implications for therapeutics and vaccine development. Cytokine Growth F R 2012; 23: 193-206.

[27] Kedzierska K, Crowe SM. Cytokines and HIV-1: interactions and clinical implications. Antivir Chem Chemother 2001; 12: 133-150. Viral induction suppression.
[28] Roberts L, Passmore JS, Williamson C, Little F, Bebell LM, Mlisana K, et al. Plasma cytokine levels during acute HIV-1 infection predict HIV disease progression. AIDS 2010; 24:819831.

[29] Wren L, Parsons MS, Isitman G, Center RJ, Kelleher AD, Stratov I, et al. Influence of cytokines on HIV-specific antibodydependent cellular cytotoxicity activation profile of natural killer cells. PLoS ONE 2012; 7:e38580.

[30] Clerici M, Shearer GM. A TH-1/TH-2 switch is a critical step in etiology of HIV infection. Immunol Today 1993; 14:107-111.।

[31] Clerici M, Sarin A, Coffman RL, Wynn TA, Blatt SP, Hendrix $\mathrm{CW}$, et al. Type 1/type 2 cytokine modulation of T-cell programmed cell death as a model for human immunodeficiency virus pathogenesis. Proc Natl Acad Sci USA 1994; 91: 1181111815.

[32] Stylianou E, Aukrust P, Kvale D, Müller F, Frøland SS. IL-10 in HIV infection: increasing serum IL-10 levels with disease progression-down-regulatory effect of potent antiretroviral therapy. Clin Exp Immunol 1999; 116:115-120.

[33] Li Y, Ling W, Xu H, Wang M, Wu C. The activation and dynamics of cytokine expression by CD4+ $\mathrm{T}$ cells and AIDS progression in HIV-1-infected Chinese individuals. Microb Pathogenesis 2012; 53:189-197.

[34] Betts MR, Nason MC, West SM, De Rosa SC, Migueles SA, Abraham J, et al. HIV nonprogressors preferentially maintain highly functional HIV-specific CD8+ T cells. Blood 2006; 107: 4781-4789.

[35] Gay C, Dibben O, Anderson JA, Stacey A, Mayo AJ, Norris PJ, et al. Cross-sectional detection of acute HIV infection: timing of transmission, inflammation and antiretroviral therapy. PLoS ONE 2011; 6:e19617.

[36] Papagno L, Spina CA, Marchant A, et al. Immune activation and CD8(+) T-cell differentiation towards senescence in HIV-1 infection. PLoS Biol 2004; 2: e20.

[37] Deeks SG, Kitchen CM, Liu L, et al. Immune activation set point during early HIV infection predicts subsequent CD4+ T-cell changes independent of viral load. Blood 2004; 104: 942-47.

[38] Regidor DL, Detels R, Breen EC, Widney DP, Jacobson LP, Palella F, et al. Effect of highly active antiretroviral therapy on biomarkers of B-lymphocyte activation and inflammation. AIDS 2011, 25:303-31.

[39] Neuhaus J, Jacobs DR Jr, Baker JV, Calmy A, Duprez D, La Rosa A, et al. Markers of inflammation, coagulation, and renal function are elevated in adults with HIV infection. J Infect Dis 2010; 201:1788-1795.

[40] Sachdeva RK, Wanchu A, Bagga R, Malla N, Sharma M. Effect of non-nucleoside reverse transcriptase inhibitors on cytokine, chemokine, and immunoglobulin profiles in serum and genital secretions of HIV-infected women. J Interferon \& Cytokine Res 2010; 30:299-310.

[41] Haissman JM, Vestergaard LS, Sembuche S, Erikstrup C, Mmbando B, Mtullu S, et al. Plasma Cytokine Levels in Tanzanian HIV-1-Infected Adults and the Effect of Antiretroviral Treatment. J Acquir Immune Defic Syndr 2009; 52:493-49.

[42] Resino S, Belloan JM, Sanchez-Ramoa AS, Gurbindo D, MuñózFernandez MA. Clinical relevance of cytokine production in HIV1 infection in children on antiretroviral therapy. Scand J Immunol 2000; 52:634-640.

[43] Moore KW, O' Garra A, de Waal Malefyt R, Vieira P, Mosmann TR. Interleukin-10. Annu Rev Immunol 1993; 11:165-190.

[44] Del Prete G, De Carli M, Almerigogna F, Giudizi MG, Biagiotti R, Romagnani S. Human IL-10 is produced by both type 1 helper (Th1) and type 2 helper (Th2) T cell clones and inhibits their antigen-specific proliferation and cytokine production. J Immunol 1993; 150:353-360.

[45] Cozzi-Lepri A, French MA, Baxter J, Okhuysen P, Plana M, Neuhaus J, et al. Resumption of HIV replication is associated with monocyte/ macrophage derived cytokine and chemokine changes: results from a large international clinical trial. AIDS 2011; 25:1207-1217.

[46] Wiercinska-Drapalo A, Jaroszewicz J, Flisiak R, Prokopowicz D. Plasma interleukin-18 is associated with viral load and disease progression in HIV-1-infected patients. Microbes Infect 2004; 6:1273-1277.

[47] Torre D, Pugliese A. Interleukin-18: a proinflammatory cytokine in HIV-1 infection. Curr HIV Res 2006; 4:423-430.

[48] Maek-A-Nantawat W, Buranapraditkun S, Klaewsongkram J, Ruxrungthum K. Increased interleukin-17 production both in 
helper T Cell subset Th17 and CD4negative T cells in human immunodeficiency virus infection. Viral Immunol 2007; 20:328.

[49] Bettelli E, Korn T, Kuchroo VK. Th17: the third member of the effector T cell trilogy. Curr Opin Immunol 2007; 19:652-657.

[50] Murray JS, Elashoff MR, Iacono-Connors LC, Cvetkovich TA Struble KA. The use of plasma HIV RNA as a study endpoint in efficacy trials of antiretroviral drugs. AIDS 1999; 13: 797-804.

[51] Nigam P, Kwa S, Velu V, Amara RR. Loss of IL-17-Producing CD8 T Cells during Late Chronic Stage of Pathogenic Simian Immunodeficiency Virus Infection. J Immunol 2011; 186:745-753.

[52] Alcaide ML, Parmigiani A, Pallikkuth S, Roach M, Freguja R, et al. Immune Activation in HIV-Infected Aging Women on Antiretrovirals - Implications for Age-Associated Comorbidities: A Cross-Sectional Pilot Study. PLoS ONE 2013; 8(5): e63804.

[53] Barqasho B, Nowak P, Tjernlund A, Kinloch S, Goh LE, Lampe F, et al. Kinetics of plasma cytokines and chemokines during primary HIV-1 infection and after analytical treatment interruption. British HIV Association. HIV Med 2009; 10:94-102.

[54] Appay V, Sauce D. Immune activation and inflammation in HIV-1 infection: causes and consequences. J Pathol 2008; 214:231-241.

[55] Grivennikov SI, Greten FR, Karin M. Immunity, inflammation, and cancer. Cell. 2010; 140:883-899.

[56] Nixon DE, Landay AL. Biomarkers of immune dysfunction in HIV. Curr Opin HIV AIDS. 2010; 5:498-503.

[57] Simonetta F and Bourgeois C. CD4CFOXP3C regulatory Tcell subsets in human immunodeficiency virus infection. Front Immunol. 2013; 4:215.

[58] Eller MA, Blom KG, Gonzalez VD, Eller LA, Naluyima P, et al. Innate and Adaptive Immune Responses Both Contribute to Pathological CD4 T Cell Activation in HIV-1 Infected Ugandans. PLoS ONE 2011; 6(4): e18779.

[59] Nagy LH, Grishina I, Macal M, Hirao LA, Hu WK, et al. Chronic HIV Infection Enhances the Responsiveness of Antigen Presenting Cells to Commensal Lactobacillus. PLoS ONE 2013; 8(8): e72789.

[60] Chevalier MF, Petitjean G, Dunyach-Remy C, Didier C, Girard P$\mathrm{M}$, et al. The Th17/Treg Ratio, IL-1RA and sCD14 Levels in Primary HIV Infection Predict the T-cell Activation Set Point in the Absence of Systemic Microbial Translocation. PLoS Pathog 2013 ; 9(6): e1003453.

[61] XIAO Jian, QIAN Ke-lei, CAO Qing-hua, QIU Chen-li, QIU Cao, XUE Yi-le, ZHANG Xiao-yan, ZHONG Ping, XU Jian-qing, L Ming-yuan and WANG Ying. HLA- DR expression on regulatory $\mathrm{T}$ cells is closely associated with the global immune activation in HIV-1 infected subjects naïve to antiretroviral therapy. Chin Med J 2011; 124(15):2340-2346.

[62] Read SW, DeGrezia M, Ciccone EJ, DerSimonian R, Higgins J, et al. The Effect of Leflunomide on Cycling and Activation of TCells in HIV-1-Infected Participants. PLoS ONE 2010; 5(8): e11937.Rey-Cuille MA, Berthier JL, Bomsel-Demontoy MC, Chaduc Y, Montagnier L, Hovanessian AG, Chakrabarti LA. Simian immunodeficiency virus replicates to high levels in sooty mangabeys without inducing disease. J Virol 1998; 72:3872-86.

[64] Rizzardi GP, Harari A, Capiluppi B, Tambussi G, Ellefsen K, Ciuffreda D, Champagne P, Bart PA, Chave JP, Lazzarin A,
Pantaleo G. Treatment of primary HIV-1 infection with cyclosporin A coupled with highly active antiretroviral therapy. J Clin Invest 2002; 109:681-8.

[65] Gilad Doitsh, Nicole L. K. Galloway, Xin Geng, Zhiyuan Yang, Kathryn M. Monroe, Orlando Zepeda, Peter W. Hunt, Hiroyu Hatano, Stefanie Sowinski, Isa Muñoz-Arias \& Warner C. Greene. Cell death by pyroptosis drives CD4 T-cell depletion in HIV-1 infection. Nature (2013).

[66] Nichole R. Klatt, Lauren A. Canary, Xiaoyong Sun, Carol L. Vinton, Nicholas T. Funderburg, David R. Morcock, Mariam Quiñones, Clayton B. Deming, Molly Perkins, Daria J. Hazuda, Michael D. Miller, Michael M. Lederman, Julie A. Segre, Jeffrey D. Lifson, Elias K. Haddad, Jacob D. Estes and Jason M. Brenchley. Probiotic/prebiotic supplementation of antiretrovirals improves gastrointestinal immunity in SIV-infected macaques. J Clin Invest. 2013; 123(2):903-907.

[67] Hontelez JA, Lurie MN, Newell ML, et al. Ageing with HIV in South Africa. AIDS 2011; 25: 1665-67.

[68] Serena Spudich and Francisco Gonza' lez-Scarano HIV-1-Related Central Nervous System Disease: Current Issues in Pathogenesis, Diagnosis, and Treatment. Cold Spring Harb Perspect Med 2012; 2:a007120.

[69] Shikuma CM, Chow DC, Gangcuangco LMA, Zhang G, Keating SM, et al. Monocytes Expand with Immune Dysregulation and Is Associated with Insulin Resistance in Older Individuals with Chronic HIV. PLoS ONE 2014 ; 9(2): e90330.

[70] Hsu et al.: Serious Non-AIDS events: Immunopathogenesis and interventional strategies. AIDS Research and Therapy 2013 10:29.

[71] Steven G Deeks, Sharon R Lewin, Diane V Havlir The end of AIDS: HIV infection as a chronic disease. Lancet 2013; 382: 1525-33.

[72] Sharon R. Lewin and Christine Rouzioux. HIV cure and eradication: how will we get from the laboratory to effective clinical trials? AIDS 2011, 25:885-897.

[73] Sandler, Netanya G and Sereti, Irini. Can early therapy reduce inflammation? Current Opinion in HIV \& AIDS January 2014 Volume 9 - Issue 1 - p 72-79.

[74] Irini Sereti, Richard M. Dunham, John Spritzler, Evgenia Aga, Michael A. Proschan, Kathy Medvik et al IL-7 administration drives T cell- cycle entry and expansion in HIV-1 infection. 2009; 113: 6304-6314

[75] Sereti I, Estes JD, Thompson WL, Morcock DR, Fischl MA, et al. Decreases in Colonic and Systemic Inflammation in Chronic HIV Infection after IL-7 Administration. PLoS Pathog 2014; 10(1): e1003890.

[76] Schackman BR, Gebo KA, Walensky RP, et al. The lifetime cost of current human immunodeficiency virus care in the United States. Med Care 2006; 44: 990-97.

[77] Bendavid E, Ford N, Mills EJ. HIV and Africa's elderly: the problems and possibilities. AIDS 2012; 26 (suppl 1): S85-91.

[78] Bor J, Herbst AJ, Newell ML, Bärnighausen T. Increases in adult life expectancy in rural South Africa: valuing the scale-up of HIV treatment. Science 2013; 339: 961-65. 